\title{
La cátedra del pensar sociológico
}

\author{
Artículos originales: SOCIOLOGÍA \\ Recibido: $15 / 09 / 2020$ \\ Aprobado: 04/11/2020 \\ Publicado: 05/02/2021
}

\section{Zenón Eduardo Vargas Morales Universidad Nacional Mayor de San Marcos zeduardovargas@gmail.com}

\section{RESUMEN}

La cátedra del pensar sociológico por su carácter crítico, desmitificador y emancipadora aporta en el análisis del carácter relacional de los procesos sociales, así como las exigencias que requiere el desempeño de su función formadora. De donde la docencia, adquiere relevancia, ya que ésta proporciona a los alumnos los conceptos teóricos, los instrumentos y técnicas para que puedan acercarse a la realidad con la competencia profesional. En ese sentido, en la primera clase, siempre quería conocer a los alumnos no solo como persona, sino principalmente acerca de su pensar sociológico y habilidades para realizar la investigación y la intervención como gestores sociales. Se les formuló a los alumnos un conjunto de preguntas para su debate en el aula: ¿qué entendían ellos por sociología como disciplina científica? ¿Qué enfoques teóricos y metodológicos habían influido más en su formación profesional? entre otras preguntas. Además se les entregó una hoja para que respondan por escrito algunas preguntas. Entonces, en el texto lo que se presenta es la dimensión cognoscitiva del pensar sociológico de los alumnos en su formación profesional.

Palabras Clave: pensar sociológico; cognición; metodología; investigación; valoración.

\section{The chair of sociological thinking}

\begin{abstract}
Due to its critical, demystifying and emancipating nature, the chair of sociological thinking contributes to the analysis of the relational nature of social processes, as well as the demands that the performance of its formative function requires. Hence, teaching becomes relevant, since it provides students with theoretical concepts, instruments and techniques so that they can approach reality with professional competence. In that sense, in the first class, I always wanted to know the students not only as a person, but mainly about their sociological thinking and abilities to carry out research and intervention as social managers. Students were asked a set of questions for their discussion in the classroom: what did they understand by sociology as a scientific discipline? What theoretical and methodological approaches had most influenced your professional training? among other questions. They were also given a sheet to answer some questions in writing. Then, in the text what is presented is the cognitive dimension of the sociological thinking of the students in their professional training.
\end{abstract}

KEYworDs: Sociological thinking; cognition; methodology; research; assessment. 


\section{Introducción}

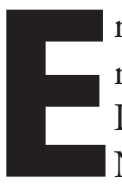
$\mathrm{n}$ un contexto de grandes acontecimientos nacionales e internacionales, en la Facultad de Letras y Ciencias Humanas de la Universidad Nacional Mayor de San Marcos, se crea el Departamento de Sociología el año de 1961 y en 1969 se convierte en una escuela autónoma en la formación de bachilleres y licenciados en la especialidad. Quince años después, el 24 de setiembre de 1984, en concordancia con la Ley Universitaria No. 23733, se promulga el Estatuto, donde se crea la Facultad de Ciencias Sociales y la Escuela Académico Profesional de Sociología. Hoy en día los alumnos egresados de pregrado pueden continuar con sus estudios de maestría y doctorado, sin necesidad de salir a otros países.

Los diversos cambios estructurales acontecidos en estos últimos 50 años en nuestra sociedad hoy en día plantean nuevos retos a la sociología. La sociología por su carácter crítico, desmitificador y emancipadora ha venido aportando en el análisis del carácter relacional de los procesos sociales, así como las exigencias que requiere el desempeńo de su función formadora. De donde la docencia, adquiere relevancia, ya que ésta proporciona a los alumnos los conceptos teóricos, los instrumentos y técnicas para que puedan acercarse a la realidad con el método científico, entendiéndose por esto, objetividad, rigurosidad, precisión desde una perspectiva crítica en el desempeño de su función profesional.

El año 2015, fue mi último año de docente que tuve a mi cargo las asignaturas de Seminario de Investigación sociológica y el de prácticas pre profesionales, en la escuela de sociología. Cuarenta años de docencia en que tuve como alumnos de las escuelas de Filosofía, Psicología, medicina, Contabilidad, el Ciclo básico en Ciencias Sociales y la Escuela de Sociología. Fueron años de grandes acontecimientos en la sociedad como: el gobierno Militar de los años 70, la presencia de los grupos subversivos y la intervención militar en la universidad en los años de 1980 y 1990 . Finalmente el retorno a la democracia y la elección de las autoridades universitarias el empezar el presente siglo. Durante los años ochenta y noventa, los jóvenes perdieron interés por la sociología, por el contexto de la violencia social y política que nos tocó vivir en las aulas. Solo recién en el presente siglo, los jóvenes que egresan de la educación secundaria, tienen interés en estudiar sociología aunque sea como una segunda opción después del derecho. A partir de esa fecha la escuela de sociología ha tenido algo más de 300 alumnos matriculados en los diez ciclos académicos.

Eran años en que la Escuela de Sociología contaba con un poco más de 30 docentes entre nombrados y contratados. La gran mayoría de los docentes estaban en la condición de principales y asociados y tenían los grados de doctor y de magíster, algunos otros con estudios concluidos en ambos grados. Docentes que habían alcanzado reconocimiento no solo como docentes, sino como investigadores en el campo académico de las ciencias sociales y algunos otros en el campo del ejercicio profesional en las diversas instituciones públicas. Que a pesar de los sueldos nada decorosos, habían puesto todo su esfuerzo y dedicación en la formación de sus alumnos que principalmente venían de los sectores populares con el entusiasmo juvenil de ser buenos profesionales; pero también, por el cariño a su Alma Mater.

En cuanto al plan de estudios, la escuela siempre organizó sus talleres de evaluación curricular cada cierto tiempo, para hacer algunos cambios en el plan de estudios, de acuerdo a los cambios que se daba con el desarrollo de las ciencias sociales y los procesos sociales en la sociedad. Casi siempre se mantenía entre 39 y 40 asignaturas dictadas en los diez semestres académicos: cursos propedéuticos en el Ciclo de Cultura General, luego cursos teóricos, metodológicos, instrumentales e informativos en la especialidad. Al concluir sus estudios los alumnos que concluían con los 220 créditos eran declarados aptos para optar el grado de bachiller de manera automática, esto hasta antes de la promulgación del nuevo estatuto. Aquí es importante destacar que en los seminarios de investigación los alumnos realizaban investigación, cuyos resultados en algunos casos se convertían en tesis y en otros casos se publicaban en revistas de la Facultad o de la Escuela de Sociología o como documentos de divulgación.

Según estudios realizados ${ }^{1}$ la mayoría de los alumnos provenían de familias que en su mayoría no eran profesionales y muy pocos con estudios superiores, pero en áreas que no tenía ninguna relación con las ciencias sociales. Precisamente, por ello, para la mayoría de los alumnos la sociología fue una segunda

1 Vargas Morales, Z.E. Sociología, universidad y sociedad en tiempos de conflicto. Inédito por publicarse. 2019. 
opción. Ellos mismos señalaban que ingresaron por cuanto el puntaje para sociología era más bajo que para ingresar a la Facultad de Derecho. Por eso, al concluir sus estudios de Cultura General, siempre optaron por trasladarse, algunos con éxito y otros se vieron obligados a continuar con sus estudios y gracias a sus docentes fueron encontrando el cariño por esta profesión.

El testimonio de uno de los alumnos: "Quería estudiar derecho, quería ser marino mercante, quise ser de todo porque consideraba que mi autosuficiencia y mi voluntad serian necesarias para ser alguien en la vida. Aún sigo preguntándome ¿cómo llegué a estudiar sociologia? Fue un enorme muro de prejuicios, y valoraciones que solo cuestionaban mi decisión. Me molestaba, me incomodaba y sentí hasta vergüenza contarle sobre la sociología a la gente, hasta que decidi tener una definición propia, concreta y resumida para aquellos que me preguntaran, y evitaba ahondar en el tema. Todo ese malestar se me pasaba cuando en clases de teoría sociología estudiábamos los aportes teóricos de sociólogos como Weber, Durkheim, o el mismo Marx, con una interpretación de los temas sociales extraordinaria y quedaba nuevamente maravillado y orgulloso de la sociologia» (Luis Angel).

Otro alumno señala por qué terminó estudiando sociología. "Debo reconocer que hubo una feria en los colegios ofreciendo carreras profesionales induciendo lo dificil que era postular a universidades de renombre. Ser político no pasaba por mi cabeza, quizá el estudiar la carrera derecho esté orientado a mis inclinaciones de defensor, no sabia de la existencia de la carrera de sociologia (...). Siempre quise ser sanmarquino, se hablaba tan bien de esta universidad que necesitaba ser parte de ella para cambiar aquel sentido de frustración de mis padres. Fueron tres intentos, dos de ellos a la carrera de derecho y el tercero, me decidi por sociología, aunque antropología no dejaba de ser atractivo" (Bruno Eduardo).

La mayoría de los alumnos tenían experiencias similares, pero que con el paso de los semestres poco a poco fueron encontrando su vocación de sociólogos. Se formaron como sociólogos desde los primeros ciclos recibiendo una formación que combinó la calidad humana y una capacidad crítica y reflexiva frente a los diversos problemas y retos que planteaba la sociedad. Con una visión local y regional en lo social, económico y político. Profesionales que sepan enfrentar con calidad y eficiencia los diversos problemas y retos que demanda la sociedad.
Asimismo, es importante destacar que la Facultad de Ciencias Sociales, tenía una responsabilidad ante la sociedad, por cuanto ella nos exigía no solo formar buenos profesionales, sino conocimientos científicos socialmente válidos capaces de generar soluciones creativas en las múltiples áreas del quehacer social. Para ello, era importante que las autoridades (Decano y Director de Escuela), diseñen programas de investigación multidisciplinarias de alto nivel, proveer de una infraestructura con laboratorios y cubículos para que los grupos de estudio de docentes y estudiantes puedan desarrollar sus investigaciones, sin dejar de lado convenios con otras instituciones para tener acceso a la base de datos. Aprovechar las diversas herramientas que nos brindaba la tecnología: ordenadores, telecomunicaciones y multimedia que posibiliten un rápido intercambio de información. Se tenía que promover un cambio radical en la enseñanza universitaria, por cuanto nos encontrábamos en la «era de la información» o "la sociedad del conocimiento».

Estos diversos temas señalados como premisa, nos motivó para discutir con los alumnos del último ciclo de estudios de Sociología, acerca de los diversos temas que tiene que ver con su formación académica y profesional. Temas acerca de la sociología como disciplina científica, la sociología como práctica o intervención profesional, enfoques teóricos que les haya permitido una mejor comprensión de la sociedad en sus diversas problemáticas; autores considerados con una mayor influencia en su formación profesional y finalmente la valoración de las asignaturas y la enseñanza por los docentes.

Este artículo fue escrito para ser publicado en 2016, pero a pesar del paso del tiempo considero que aún sigue teniendo vigencia para su lectura por la comunidad de Ciencias Sociales y particularmente por los interesados en esta carrera profesional irreverente - como señalaba Bourdieu - como es la Sociología.

El término evaluar significa "dar valor» a algo, lo que, en el caso de la evaluación de los alumnos de la Escuela de Sociología a través del diálogo, consistió en obtener los saberes que tenían ellos acerca de su formación profesional. Con esta evaluación y valoración se buscó conocer la habilidad en utilizar sus conocimientos relacionados con el saber, saber hacer y saber ser, así como sus capacidades para resolver situaciones determinadas con relación a su profesión. 
Es importante señalar que la evaluación fue compleja, por cuanto tenía que ver no sólo con los planes de estudio, sino el contexto de la política académica universitaria y del país, así como los contenidos conceptuales, procedimentales y actitudinales de los docentes. En ese sentido, lo que hacemos es formular un conjunto de preguntas a los alumnos acerca de los conocimientos adquiridos relacionados con el «saber» o sea conceptuales.

\section{¿Qué es la sociología? y ¿para qué sirve la sociología?}

Son varios los autores que han tratado de responder esta pregunta, como es el caso de Bernard Lahire, quien escribe su libro con la pregunta ¿Para qué sirve la sociología?» como dice el autor es una pregunta ingenua y provocadora. ¿Debe necesariamente servir para algo la sociología? y si la sociología debe tener una utilidad, ¿cuál debe ser su naturaleza? Las respuestas han sido diversas, pero todos coinciden en señalar que por su naturaleza muy particular en cuanto a sus diversos fundamentos teóricos, metodológicos y prácticos lo ha convertido en blanco de críticas, por ser irreverente como dijera Pierre Bourdieu. Pero, es importante tener en cuenta, que la sociología nace bajo el enfoque positivista, buscando liberarse de la influencia religiosa y la especulación metafísica. En ese sentido buscó su autonomía como ciencia, a través de la objetividad en la investigación aplicada y la comprensión de los fenómenos sociales. Pero, en la historia de la sociología, desde los llamados «clásicos» hasta los contemporáneos y los sociólogos actuales, se han desarrollado diversos enfoques teóricos y metodológicos sumamente rigurosos en la explicación y comprensión de la sociedad, al igual que cualquier otra ciencia. Para esto, emplea rigurosos métodos cuantitativos y cualitativos y como resultado del análisis llegar a la comprensión, la reflexión acerca de las interrelaciones entre distintos campos o áreas de los fenómenos sociales.

\section{La sociología como disciplina cientifica}

$\mathrm{Al}$ grupo de alumnos que terminaban el último año de estudios de sociología, la primera pregunta que se les formuló para el diálogo fue: ¿Cuál era la compren- sión que tenían acerca de la sociología como disciplina científica? Responder a esta pregunta, no fue fácil para los alumnos. Cuando uno pregunta ¿qué es la sociología? Muchos contestaron diciendo que es: «la ciencia que estudia la sociedad». Posiblemente es una respuesta que no se puede refutar, pero tampoco no explica nada y por lo tanto no convence a nadie, menos a la gente común que siempre nos interroga. Lo que se apreciaba en cada una de las respuestas de los alumnos era precisamente eso. De hecho, se continuó con más preguntas de acuerdo a las respuestas, como: ¿qué es la sociedad? ¿Qué es la realidad social? ¿Qué son las relaciones sociales?, ¿qué son los fenómenos sociales? Las respuestas a cada una de estas preguntas, tampoco eran convincentes y no conceptualizaban relacionándola con una temática o campo concreto de análisis o de estudio.

Aquí algunas de las respuestas de los alumnos, frente a la pregunta: ¿Cuál es su comprensión acerca de la sociología como disciplina científica?

- Es la ciencia que se encarga del estudio de la sociedad.

- Busca comprender la realidad social

- Ciencia que estudia los problemas que ocurren en la sociedad.

- Se encarga del estudio de las relaciones sociales en sus diversos niveles y formas.

- Entender los diferentes fenómenos sociales desde diferentes ópticas.

- Estudia el comportamiento, las acciones de los individuos y fenómenos sociales.

- Estudia los fenómenos sociales, como la acción social, dinámica social e intersubjetividad.

- Produce conocimientos especificos que puede ser útil para la sociedad.

- Estudia en forma explicativa y comprensiva la sociedad.

- Estudia las relaciones sociales entre los individuos.

- Estudia las relaciones sociales en su aspecto micro y macro.

- Busca las regularidades entre las relaciones humanas a nivel grupal.

- Estudia las relaciones entre el hombre y la sociedad.

- Estudio metódico y sistemático de las relaciones sociales.

La sociología aspira, desde sus orígenes, a ser una ciencia que busca explicar la sociedad, los fundamentos de la convivencia social. Es decir, es una rama del conocimiento humano que tiende hacia la compren- 
sión racional y objetiva de un ámbito de la realidad, no sólo por los científicos sociales, sino principalmente por la misma población, con la finalidad de que orienten su vida en base a conocimientos objetivos resultado de la investigación. Según Salvador Giner (1999), "la sociología es una disciplina empírica, teórica, abierta, éticamente reflexiva y socialmente crítica» ${ }^{2}$.

La sociología es la clave de la cultura intelectual moderna ya que vivimos en un mundo enormemente preocupante, pero que presenta las más enormes esperanzas para el futuro. El mundo contemporáneo es totalmente diferente al del pasado y la labor de la sociología es comprender este mundo y lo que puede presentarse en el futuro. Un sociólogo es un analista, describe y cuestiona acerca de temas que afectan nuestras vidas. El carácter crítico de la sociología le permite develar los problemas y contradicciones de fondo que existen en las sociedades modernas, así como mostrar caminos para solucionar y, de esta manera, contribuir realmente a mejorar las condiciones y calidad de vida de las personas y comunidades.

Efectivamente, desde su nacimiento la sociología como disciplina siempre tuvo diversas conceptualizaciones. Según Durkheim la sociología consiste en captar, entender y medir los hechos humanos como si fueran cosas. Weber por su lado, afirma que «la sociología es una ciencia que pretende comprender, interpretándola, la acción social (la acción de sujetos que se dirigen hacia la conducta de otros sujetos) para, de esa manera, explicarla causalmente en su desarrollo y efectos», o de Alan Touraine, para quien la sociología tiene por objeto explicar y comprender las conductas de los actores sociales, mediante el análisis de las relaciones que mantienen entre sí. Anthony Giddens, en su libro "en defensa de la sociología», señala que «la sociología tiene algo que provoca una irritación que no logran suscitar otras disciplinas académicas» ${ }^{3}$. Agrega que la sociología «es una disciplina generalizadora que se ocupa principalmente de la modernidad: del carácter y la dinámica de las sociedades modernas o industrializadas». Y concluye afirmando que "de todas las ciencias sociales la sociología, es la que trata más directamente de las cuestiones que nos afectan en nuestra vida cotidiana; el desarrollo del urbanismo

2 Salvador Giner, Sociología, Nueva Edición Revisada, 1999.

3 Anthony Giddens, en defensa de la sociología, traducción de Jesús Albores, Alianza Editorial, 2000. moderno, el crimen y el castigo, el género, la familia, la religión, el orden social y económico» (Giddens 2000: 14), finalmente, Karl Marx (1818-1883) puso en el centro de la preocupación sociológica los conflictos sociales entre clases en la sociedad capitalista, las diferentes formas de alienación humana, los procesos históricos y la emancipación como principio y aspiración de superación de toda forma de dominación del hombre. Los teóricos de la Escuela de Frankfurt definían a la sociología como una ciencia que busca develar la realidad oculta, hacer visibles los sistemas ocultos de dominación en la sociedad. En ese sentido, son tantas las definiciones que existen acerca de lo que es la sociología. Pero, la importancia está en saber reconocer el contexto histórico y específico en el que se pretende no solo definir, sino plantear soluciones a cualquier problema que afectan la vida principalmente de los más oprimidos de la sociedad. Un pensamiento social que busque cambiar una sociedad neoliberal que busca construir un orden social basado en el individuo, considerado como un ente abstracto, limitado a relaciones de tipo transaccionales. Una dominación neoliberal que busca someter las relaciones sociales al mercado.

Pues, en la actualidad, estamos viviendo en una «sociedad de individuos» dispersos que no está basada en la solidaridad colectiva, ni agrupados en partidos políticos, sino en la responsabilidad o autorresponsabilidad individual.

\section{La sociología como práctica e intervención profesional}

Otra pregunta abierta para dialogar con los alumnos fue acerca de la sociología como disciplina científica y como práctica y la interrelación entre ambas. Teniendo en cuenta que esta dicotomización había sido una de las divisiones más persistentes en la sociología. La que distingue entre investigación aplicada e investigación básica. La primera se consideraba como aquella que respondía a un objetivo utilitario, mientras que la segunda es la que tenía como cometido contribuir al conocimiento de la realidad, de las relaciones sociales entre los individuos. La división estuvo sometida a importantes polémicas y críticas debido a las inconsistencias que presentaban cuando se atribuía características distintivas al trabajo científico o a los conocimientos que genera. 
Por sociología aplicada como práctica se suele entender la tarea sociológica orientada a la resolución de problemas prácticos que afectan nuestra vida cotidiana. El término se utiliza de manera equivalente a investigación social aplicada cuando se establecen diferencias respecto a la investigación dirigida principalmente a aportar conocimientos sobre la realidad social y la búsqueda de la verdad. No obstante, en el caso de la sociología, este asunto ha sido especialmente problemático y aún hoy sigue estando sujeto a cierta controversia. De un lado, el término sociología aplicada se emplea con bastante ambigüedad, con lo que ocurre algo parecido a lo que Robert Merton llamaba un "problema de fijación de hechos" ${ }^{4}$ (Merton, 1992). En ocasiones el término no se hace explícito $y$, a veces, existen dificultades para saber a qué parcela de la tarea sociológica nos referimos cuando hablamos de sociología aplicada: ¿estamos hablando de investigación orientada por problemas sociales?, ¿de investigación que pretende tener utilidad en el ámbito público o privado?, ¿¿de investigación empírica o de aplicación de ciertas técnicas de investigación? De otro lado, esa falta de fijación tiene algunas consecuencias prácticas que dan lugar a cierta ambivalencia a la hora de valorar los resultados de las investigaciones. Por ejemplo, ¿qué carácter tienen los conocimientos que resultan de las investigaciones aplicadas? ¿'Si una investigación es aplicada sus resultados deben ser valorados desde un criterio similar a los de la investigación básica?

Hay que señalar con claridad, que la diferencia entre la investigación básica y la intervención transformadora sobre la realidad, siempre se ha sostenido que la investigación básica es instrumental a la transformación social a través de la intervención sociológica; es decir, que la investigación básica sirve o contribuye a la transformación, pero no lo realiza el investigador, porque no es su función específica. La diferencia está en que el primero es instrumental, para el mejor ejercicio profesional del que hace la transformación de la realidad o el diseño de políticas públicas del Estado o la empresa privada.

A los alumnos se les formuló la pregunta siguiente: ¿Cuál es la comprensión que tienen acerca de la sociología como práctica e intervención profesional?

4 Merton Robert K. Teoría y estructuras sociales, FCE, México, 1992.
Cada una de las respuestas de los alumnos se agrupa en el siguiente cuadro resumen:

Es cuando la sociología pone su mirada cientifica en aquellas personas que no son visualizados por las autoridades ofuncionarios públicos.

Es cuando el sociólogo busca cambiar la sociedad, haciendo uso del conjunto de conocimientos recibidos en la universidad.

Analiza, evalúa y pone en práctica sus conocimientos para resolver algún problema social especifico o de la sociedad en general.

Ejerce la profesión, interviniendo en la solución de los problemas sociales que permita mejores condiciones de vida de la población.

Consiste en hacer diagnósticos y analizarlos criticamente, que sirvan para el diseño de políticas públicas locales $o$ regionales.

La sociología, desde que va definiendo su propia identidad, bajo presupuestos epistemológicos del positivismo, siempre buscó recabar suficiente conocimiento sobre la sociedad como para establecer una serie de leyes que permitiesen el control de los fenómenos sociales. Hacían esto, animados por los avances experimentados en las ciencias naturales. Montesquieu se inspiró en el concepto de ley acuñado por Newton para tratar de aplicarlo de igual forma a los fenómenos sociales. Se entendía que una vez comprendidas las leyes básicas del funcionamiento de la sociedad, sería posible organizar dicha sociedad sobre una base científica y racional ${ }^{5}$.

Los sociólogos, al igual que otros profesionales de las ciencias sociales, tienen que tener en cuenta que la reflexión y comprensión de la complejidad del campo social es el eje irrenunciable de nuestra formación como sociólogos. Mi experiencia como profesional y académico en las asignaturas de investigación y de prácticas preprofesionales, me permitieron entrar en contacto con profesiones de otras disciplinas como psicólogos, economistas, trabajadores sociales, antropólogos, ingenieros, médicos, geógrafos que trabajan en diferentes instituciones y comprender que hoy en día existe la necesidad de un trabajo inter y multidis-

5 Saco Alvarez, Alberto. Sociología aplicada y cambio social. http:// ctinobar.webs.ull.es/1docencia/Cambio\%20Social/SACO.pdf 
ciplinario. Incluso los profesionales de las áreas médicas e ingenierías toman en cuenta que lo social lo impregna todo, de donde hasta la construcción de una carretera pasa por un previo análisis de las condiciones socioeconómicas y culturales de los pueblos por donde ha de pasar la vía terrestre, de ello se desprende la necesidad de una previa intervención sociológica. Cuando desde el estado se busca promover el cambio social para mejorar la calidad de vida de la población, individual y/o colectiva exige de una intervención profesional especializada y multidisciplinaria, donde el papel del sociólogo es fundamental.

El debate en cuanto a la sociología teórica y aplicada, o en otros términos entre el enfoque ingenieril frente al enfoque clínico sigue abierto. Los Congresos de sociología han sido espacios de debate, señalando peyorativamente que los que trabajan para el sistema de dominación aún vigente, hacen sociología aplicada y "cínica», y por otro lado, los «no cínicos» hacen una verdadera sociología crítica. Pero, resulta que hoy en día tal separación no tiene sentido, por cuanto existe una dialéctica, donde la práctica se sustenta en una teoría científica y la teoría no puede existir sin sustento práctico. Posiblemente son muchos los partidarios de una sociología práctica y aplicada, más vinculada a objetivos políticos o normativos, como también aquellos que creen que la sociología debe estar dedicada única y exclusivamente a producir conocimiento social independientemente de sus posibles aplicaciones, postura principalmente de aquellos sociólogos que exclusivamente se han dedicado a la vida académica y nunca tuvieron la experiencia profesional en otras instituciones no académicas. Pues los sociólogos son legitimados o deslegitimados, de acuerdo a la función que realizan como profesionales o según la utilidad que se le asigne socialmente a los resultados de la investigación que realizan. En ese sentido, para algunos la sociología es útil porque es capaz de hacer diagnósticos y análisis de la sociedad. Mientras que otros deslegitiman, por considerar como una profesión que lucha a favor de los oprimidos y contra del poder. Incluso otros afirman que es una disciplina que genera instrumentos para la dominación de quienes ejercen el poder político. Como también hay aquellos que consideran que es una disciplina científica, cuyos fundamentos teóricos, permiten desarrollar proyectos de cambios sociales a través de la intervención o participación en las políti- cas públicas del gobierno regional, local o nacional, a través de una mediación con las comunidades.

Recogiendo la postura de muchos profesionales, podemos afirmar que la sociología aplicada no siempre está al servicio de la dominación, como afirman los sociólogos que asumen una postura teórica crítica. Sin una postura crítica no puede haber cambios en las estructuras de dominación, a partir de nuestra participación como interventores en las políticas públicas. En la actualidad el profesional sociólogo tiene mucha demanda en la sociedad como consultor o asesor de organismos públicos, empresas, organizaciones del más diverso tipo, jugando un papel destacado como suministrador de información para la toma de decisiones. De donde, tiene una gran relevancia el vínculo entre la formación académica y profesional en sus cuatro grandes bloques, es decir, el teórico-conceptual, el metodológico, el técnico instrumental y el conocimiento del proceso histórico de la sociedad, además de la adquisición de experiencias en espacios laborales que retroalimenten la vida académica desde una perspectiva crítica.

En conclusión, los alumnos tenían claridad en cuanto al rol del profesional sociólogo, que puede desempeñar tres roles a la vez o separadamente, o los tres al mismo tiempo. Por un lado, está el rol ideológicopolítico, donde la mayoría de los sociólogos asumen una postura crítica frente a los diversos aspectos de la sociedad y plantean alternativas de solución. Este rol ha tenido cambios con el transcurso en el modo de ser de la sociedad, y las discusiones siempre han girado en torno a la estructura social, la dinámica de las clases sociales, el problema del sistema democrático, así como la crisis de las instituciones, la clase política y la crítica al modelo neoliberal de los gobiernos. Por otro lado, el sociólogo es un investigador, que recoge y analiza diversos datos, para comprender y explicar críticamente el porqué de los problemas que afectan a la sociedad, ya sea en parte o en su totalidad, de esta manera contribuye al conocimiento de la sociedad y de la vida. Hoy en día el sociólogo, cada vez más tiene interés por el conocimiento científico de la sociedad, en una época signada por la globalización y las poderosas influencias de la tecnología, la información y la comunicación; hacen que sus instrumentos teóricos y metodológicos sean afinadas acorde a los tiempos, para que no queden dudas acerca de la confiabilidad de sus hipótesis y resultados. Se demanda del sociólo- 
go sustentar científicamente sus afirmaciones, con la finalidad de contribuir al mejoramiento de un trabajo técnico para la formulación y evaluación de proyectos de desarrollo humano multidimensional. Así como diagnosticar de manera rápida diversas situaciones socio-económicas. En tercer lugar, el sociólogo hace el rol de «ingeniería social», planificando, haciendo diseños de políticas públicas sociales, sustentado en sus conocimientos sociológicos; Gerencia social y gestión, interviniendo y proponiendo alternativas más racionales y críticas frente a algunos aspectos de la realidad que se desea modificar o cambiar. A través del desempeño de estos roles, el sociólogo ha ido construyendo su identidad profesional. Bourdieu en su libro «Lección sobre la lección, afirma que el rol del Sociólogo en la sociedad no es cumplir felizmente con las normas, valores y políticas de las instituciones o adscribirse fácilmente a uno o a otro grupo de opinión y presión social, sino el rol del Sociólogo es el de mostrar las contradicciones y fenómenos de la comunidad, no observables a simple vista o no explicitadas en el lenguaje y la conversación pública, e invitar tanto a unos como a otros a reflexionar sobre sí mismos y sus determinantes sociales y culturales (Bourdieu, 2002).

\section{Enfoques teóricos y problemas sociales hoy}

¿Cuál es el enfoque teórico y metodológico que les ha permitido comprender y explicar mejor los problemas sociales de hoy? Esta pregunta se les planteó a los alumnos bajo la premisa (no digo hipótesis) de que para el sociólogo la teoría sociológica nos permite ver los aspectos ocultos de la realidad social. Pero, no de cualquier manera, sino, tiene que ser sistemática, coherente guiado por un adecuado manejo metodológico y conceptual. Por eso, la sociología se tiene que definir en relación a la teoría sociológica. La teoría es lo que nos permite el manejo adecuado de conceptos y métodos, como también nos permite comprender qué cosas son relevantes, como también podemos evitar perdernos haciendo preguntas no relevantes o sin sentido. Hugo Cerda (1998) y Roberto Hernández Sampieri (2003), señalan que la teoría tiene como función: prevenir errores, orientar la realización del estudio, ampliar el horizonte del estudio, documentar la necesidad de realizar el estudio, producir nuevos conocimientos, proveer de un marco de referencia para explicar la realidad. De otro lado, se argumenta que una buena teoría es aquella que es capaz de describir, explicar y adelantar predicciones sobre el objeto de estudio ${ }^{6}$. Aquí las respuestas de los alumnos:

Todos los enfoques teóricos de las ciencias sociales en general y de la sociología en particular son válidas para comprender los problemas sociales.

Para los que estamos interesados en explicar los problemas sociales que se dan en la vida cotidiana, por su puesto es el interaccionismo simbólico

Enfoque cultural. Por cuanto tiene mucha influencia en el desarrollo económico social. Podemos proyectarnos desde lo global hasta las estructuras básicas administrativas en una institución.

El enfoque de la sociología comprensiva de Max Weber, sigue vigente en la comprensión de los problemas sociales que se observa a diario.

La teoría de la estructuración. Tenemos que tener en cuenta que todas las personas otorgan sentido a lo que ellos y otros hacen. En esa medida, los significados son producidos y reproducidos vía aplicación práctica como dice Giddens.

La dialéctica de Carlos Marx. Es un enfoque por excelencia dentro de las ciencias sociales, por ser un pensamiento y un método más racional y adecuado no sólo para comprender los problemas, sino para transformar el mundo. Nos permite una mayor libertad de critica.

La etnometodología y el interaccionismo simbólico. Considero que el mundo globalizado nos exige un análisis mucho más sutil y crítico de los significados de esta vida cotidiana cada vez menos comprendida por los cientificos sociales.

Los enfoques de Bourdieu. Él maneja un conjunto de conceptos como el de campo, habitus, violencia simbólica, poder simbólico, capital, entre otros, muy útiles como teoría y método en la comprensión del mundo actual.

Estas respuestas grafican una formación teórica metodológica muy plural, por cuanto los alumnos expresan su mayor identificación con ciertos enfoques o perspectivas teóricas en el análisis e investigación de temas sociológicos. En los cursos que tienen que ver con los enfoques teóricos a los alumnos se les ha proporcionado un conocimiento profundo de

6 Hernández Sampieri Roberto. Metodología de la investigación, Mc Graw Hill, 5ta. Edición, 2010. 
las principales teorías sociológicas contemporáneas y las tendencias teóricas, metodológicas y temáticas presentes en desarrollo más reciente de la teoría sociológica. Partiendo de la teoría de Max Weber, la «sociología hermenéutica del conocimiento» que recibió su fundamentación fenomenológica por medio de Schutz y su perfil como sociología del conocimiento por medio de Berger y Luckmann. Desde la perspectiva históricas, las propuestas de Wallerstein y el marxismo analítico, pasando por la sociología de Bourdieu, Giddens, Luhmann, Castells, Bruno Latour, Sygmund Baumann, Maffesoli y la sociología histórica de Skópol, Barrington Moore y Arato, entre otros, aunque estos últimos están ausentes en las respuestas de los alumnos.

En los Talleres y Seminarios de Investigación, se exigía a los alumnos investigar los problemas sociales, desde la construcción teórica del objeto de estudio y su ejecución hasta culminar con la comunicación de los resultados. Todo esto, desde diversas tendencias de la sociología contemporánea, analizando la vigencia actual del pensamiento clásico, y las perspectivas teóricas actuales que los estudiantes libremente asumen como opción teórica y que es motivo de debate en las aulas.

Además de puntualizar que la reflexión, el pensamiento, acerca de la vida social de los hombres es anterior a la constitución de la sociología como ciencia. Cuando uno lee los escritos de Platón y Aristóteles, nos dan cuenta su preocupación sobre ¿̇cómo debería de ser una sociedad buena? Esta pregunta recorre toda la historia de la humanidad, pasando por Santo Tomás, los socialistas utópicos, Marx, y hoy todos los científicos sociales, aspiramos a ello: ¿cómo hacer una sociedad buena, con equidad, libre, justa, democrática y bella?

\section{Autores con mayor influencia en la formación profesional de los alumnos}

Es desde esa opción o preferencia, que los alumnos se identificaban mucho más con ciertos autores ya sean clásicos o contemporáneos. Aquí era importante que señalen autores clásicos y contemporáneos que mayor influencia habían tenido en su formación profesional. Esta información se solicitó con el conocimiento de que los alumnos de sociología habían analizado como parte de su formación teórica desde Saint Simon, pa- sando por Comte, Durkheim, Weber, Marx, hasta las propuestas teóricas actuales.

Cabe mencionar que en el diccionario enciclopédico de las Ciencias Sociales se señala que «a la sociología Comte sólo le proporcionó el nombre». Esta afirmación parte de que Augusto Comte en su condición de secretario personal del Conde Claude Henri Saint Simon, habría plagiado de las enseñanzas recibidas del Conde. Bueno, hablar de cada uno de los autores necesitaría muchas páginas y tiempo, que no es el caso ahora.

Las respuestas de los alumnos del último Ciclo son como sigue:

\begin{tabular}{|l|c|l|c|}
\hline $\begin{array}{c}\text { Autores que más } \\
\text { han influido en su } \\
\text { formación profesional }\end{array}$ & N.o & $\begin{array}{c}\text { Autores que más } \\
\text { han influido en su } \\
\text { formación profesional }\end{array}$ & N.o \\
\hline Max Weber & 15 & Talcott Parsons & 3 \\
\hline Pierre Bourdieu & 10 & Berger y Luckman & 3 \\
\hline Irving Goffman & 9 & Michel Foucault & 2 \\
\hline Emili Durkheim & 8 & Jurgen Habermas & 2 \\
\hline Alfred Schutz & 7 & Robert Merton & 2 \\
\hline Carlos Marx & 5 & George Simmel & 2 \\
\hline Anthony Giddens & 4 & Ulrich Beck & 1 \\
\hline Mead & 4 & Augusto Comte & 1 \\
\hline Garfinkel & 4 & Niklas Luhmann & 1 \\
\hline
\end{tabular}

Pregunta formulada a los alumnos del Seminario de Investigación Social, Escuela de Sociología. Agosto, 2015. UNMSM.

En las respuestas de los alumnos que se encuentra en el X Ciclo, se observó que los autores que más habían influido en su formación teórica y metodológica eran: Weber, Bourdieu, Durkheim, Schutz y medianamente Marx. Definitivamente esto se debía al mayor dominio teórico e incluso preferencia de los profesores que enseñaban el curso de teoría sociológica o los Seminarios de investigación. Como se puede observar, hay coherencia con el cuadro anterior, donde autores como Baumann, Lipovesky y Latour están ausentes, asimismo, Wallerstein y Castells que tienen una mirada más global e histórica de la sociedad. En conclusión, los alumnos recibieron con mayor énfasis enfoques como la sociología comprensiva, la etnometodología y el interaccionismo simbólico, la fenomenología y una sociología crítica y dialéctica 
sin mayor influencia en cada uno de ellos. Estos autores son sus principales referentes teóricos con el cual egresaron como sociólogos para ejercer su profesión de sociólogo.

Páginas de «sociólogos.com», recoge el estudio realizado por "Amazon" y datos de "ISI Thomson Reuters», donde se afirma que "la Sociología está de moda, pero los sociólogos no. La Sociología es una de las disciplinas de mayor actualidad y uso científico como lo demuestra la enorme venta de libros sobre temas sociales y la elevada citación de autores de Sociología en revistas científicas. Sin embargo, la profesión de sociólogo afronta problemas de identidad, inestabilidad laboral y desconocimiento externo» ${ }^{7}$.

Se afirma en el informe "que existan best-sellers sobre temas sociales demuestra un marcado interés de la población por el estudio del comportamiento social, entender la conducta humana y analizar las relaciones sociales. Es decir, por los asuntos tratados por la Sociología. En el ranking de los libros más vendidos en la década del 2000 no aparecen libros de política, ni de finanzas, ni de economía, ni de medicina. ¿Por qué la gente cuando compra un libro se interesa por las relaciones y el comportamiento social? Tal vez para ganar seguridad, mejorar su vida social o simplemente porque es más entretenido. Sea la razón que fuere, es innegable que a la sociedad le interesa documentarse y aprender sobre temas sociales como cambio social, empatía, relaciones o redes. La Sociología lleva mucho tiempo estudiando y dando nombre a estos temas».

"Otro indicador que señala la buena salud de la Sociología es la amplia citación de autores de Sociología. Entre los textos más citados en las humanidades y las ciencias sociales se encuentran principalmente libros y artículos de Sociología. El análisis de los autores de libros más citados en 2007 en la rama de las Humanidades, basado en datos de ISI Thomson Reuters, es revelador: 7 de los 10 autores más citados en artículos científicos y libros en Humanidades son sociólogos: Foucault, Bourdieu, Giddens, Goffman, Habermas, Weber y Latour (ver tabla). El resto de autores en el ranking de los más citados en Humanidades lo completan dos filósofos, Derrida y Butler, y un psicólogo, Bandura. Aunque

7 http://ssociologos.com/2015/01/29/la-sociologia-esta-de-modapero-los-sociologos-no/ y datos de ISI Thomson Reuters
Foucault y Habermas también pueden ser considerados filósofos, la preponderancia de la Sociología sobre otras ciencias es manifiesta a la hora de hacer investigación. Para construir conocimiento científico se usan teorías de la Sociología como el poder (Foucault 1977), tipos de capitales (Bourdieu 1986), habitus (Bourdieu 1984), estructuración (Giddens 1984), representaciones (Goffman 1959), acción comunicativa (Habermas 1989), empatía (Weber 1922), o actor-red (Latour 1987)» ${ }^{8}$.

Autores más citados en Ciencias Sociales y Humanidades en 2007

\begin{tabular}{|l|l|r|}
\hline Autores & Disciplina & Citas a libros \\
\hline Michael Foucault & Filosofía, Sociología & 2,521 \\
\hline Pierre Bourdieu & Sociología & 2,465 \\
\hline Jacques Derrida & Filosofía & 1,874 \\
\hline Albert Bandura & Psicología & 1,536 \\
\hline Anthony Giddens & Sociología & 1,303 \\
\hline Irving Goffman & Sociología & 1,066 \\
\hline Jürgen Habermas & Sociología, Filosofía & 1,049 \\
\hline Max Weber & Sociología & 971 \\
\hline Judith Butler & Filosofía & 960 \\
\hline Bruno Latour & Sociología, Antropología & 944 \\
\hline
\end{tabular}

Fuente: Thomson Reuters ISI, web of Sciences. Extraído de timeshighereducation.co.uk

Se concluye señalando que «tanto para el consumo masivo como para la creación de ciencia se recurre a la Sociología. La Sociología está de moda. Sin embargo, a pesar de la popularidad científica y social de la Sociología, la profesión de sociólogo afronta graves problemas: desconocimiento externo a qué hace un sociólogo, inestabilidad laboral y falta de identidad».

\section{Valoración de los cursos para su formación profesional.}

Señale las asignaturas que han sido decisivos en su formación profesional como sociólogo. Aquí es importante destacar que todas las asignaturas son parte del plan de estudios que en su momento fueron diseńados de acuerdo al perfil profesional señalado en el Plan de Estudios de la escuela, como también según las exigencias del mercado ocupacional. La

8 http://ssociologos.com/2015/01/29/la-sociologia-esta-de-modapero-los-sociologos-no/ y datos de ISI Thomson Reuters. 


\begin{tabular}{|l|c|l|l|}
\hline \multicolumn{1}{|c|}{$\begin{array}{c}\text { Asignaturas que han sido decisivas en su formación } \\
\text { profesional }\end{array}$} & No. & \multicolumn{1}{|c|}{$\begin{array}{c}\text { Asignaturas que han sido decisivas en su formación } \\
\text { profesional }\end{array}$} & No. \\
\hline Teoría Sociológica & 11 & Sociología Rural & 2 \\
\hline Seminario de Investigación social & 10 & Planificación Social & 2 \\
\hline Sociología de la Cultura & 9 & Antropología General & 1 \\
\hline Métodos y técnicas de Investigación Sociológica & 6 & Estructura y Lógica de la Investigación Científica & 1 \\
\hline Sociología Política & 5 & Política Social & 1 \\
\hline Metodología de la Investigación Sociológica & 3 & Introducción a la Sociología & 1 \\
\hline Sociología del Desarrollo & 3 & Literatura y Sociedad & 1 \\
\hline Intersubjetividad & 3 & Sociología de las Organizaciones & 1 \\
\hline Sociología de Género & 2 & Demografía social & 1 \\
\hline Sociología del Trabajo & 2 & Filosofía & 1 \\
\hline Sociología Urbana & 2 & & \\
\hline
\end{tabular}

Pregunta formulada a los alumnos del Seminario de Investigación Social, Escuela de Sociología. Agosto, 2015. UNMSM.

baja puntuación en algunas asignaturas se debe no a la poca importancia del curso, sino que los docentes responsables en el dictado del mismo, no supieron desarrollar a satisfacción y expectativa de los alumnos.

Según la respuesta de los alumnos, el curso de teoría sociológica fue considerado decisivo en su formación profesional, por cuanto es a través de los clásicos y contemporáneos que les había permitido mirar sociológicamente esta compleja realidad social. Aquí tiene toda una importancia el papel de los docentes para enseñar a pensar desde estos autores una realidad distinta a la que fue el referente de reflexión de cada uno de estos autores. Por otro lado, el curso de Seminario de Investigación Social, también consideraron decisivo en su formación, por cuanto es a través de esta asignatura que los alumnos empezaron a formarse como investigadores. De hecho, para los alumnos, formular preguntas puntuales a la sociedad no fue fácil. De donde, los alumnos tenían que aprender a dar respuesta a los problemas que se formula, haciendo uso del conjunto de conocimientos teóricos, metodológicos e instrumentales que habían recibido a lo largo de su formación académica. Los demás cursos, no habían tenido mayor peso en la consideración de cada uno de los alumnos.

Las asignaturas que integran la disciplina se impartía desde el primer Ciclo de estudios hasta el décimo semestre académico. Cursos propedéuticos en los dos primeros años y cursos de especialidad que tenían que ver con la formación básica desde las cuestiones teóricas, epistemológicas, instrumentales y de conocimiento de la realidad peruana y latinoamericana.
Agregándose cursos que tenían que ver con las habilidades del estudiante en cuanto a la investigación y la intervención y práctica de la profesión.

Aquí se puede observar que ciertos cursos como demografía, geografía, lenguaje, estadística no eran muy valorados por los alumnos. Definitivamente, cada uno de los cursos señalados era importante como instrumento de análisis, pero a decir de los mismos alumnos, el problema está en los docentes que tienen limitaciones didácticas y metodológicas en la trasmisión de los temas a tratar. Afirmaban que algunos docentes exageraban en el uso de las diapositivas o los videos, en vez de un análisis y debate de los temas contenidos en cada una de las asignaturas.

En cambio los Seminarios de investigación tenían una mayor valoración por parte de los alumnos, debido a que metodológicamente, se diversificaba la enseñanza a través de conferencias, seminarios, exposiciones, diapositivas, videos y clases prácticas, poniendo en debate, no sólo lecturas de los clásicos y contemporáneos, sino que principalmente se analiza las investigaciones realizadas por los sociólogos peruanos desde diversas perspectivas teóricas y metodológicas, contextualizando su estudio y sometiéndolo a un análisis crítico. Esta forma de enseñanza, unido a la autopreparación de los estudiantes contribuyó a desarrollar las competencias teóricas y habilidades propias para su desempeño profesional.

El resultado final consistió en la presentación de un avance de investigación que finalmente sea la base para la tesis de licenciatura. En los seminarios, los alumnos desarrollan un conjunto 
de saberes, que a su vez tenía que traducirse en $s a-$ ber hacer y finalmente, el saber ser de los alumnos.

Otra pregunta que orientó el debate con los alumnos del Seminario fue acerca de: ¿Qué recomendaciones haría usted, para mejorar la formación profesional de los alumnos? Era importante que los alumnos sugieran aspectos a tener en cuenta para mejorar la enseñanza de los alumnos de sociología. Aquí las respuestas:

Un mayor control del Plan de estudios, para evitar la repetición de temas en varios cursos.

Ampliar el curso de Métodos y Técnicas de Investigación.

Que la teoría esté acompañada del análisis de los problemas prácticos que se dan en la sociedad.

Que los docentes mejoren su pedagogía y didáctica en la enseñanza de cada una de las asignaturas.

Introducir en el plan de estudios cursos de idiomas como el inglés, el quechua.

Implementar prácticas de salida y de contacto con la realidad desde los primeros años.

En el Ciclo de formación general se debe incluir más cursos de carrera o especialidad

Implementar las prácticas pre profesionales desde el tercer año.

Formar grupos de estudio a través de talleres y Seminarios.

Hacer una mejor selección de los jefes de práctica.

Motivar a los alumnos para que asuman con responsabilidad sus estudios profesionales.

Poner mayor énfasis en los cursos instrumentales que nos permita competir con los que egresan de otras universidades.

Reestructurar el Ciclo de formación general en cuanto al plan de estudios y la designación de docentes que sean los más preparados.

Implementar cursos de informática, para estudiar programas como el Atlas Ti y el SPSS.

Pregunta formulada a los alumnos del Seminario de Investigación Social, Escuela de Sociología. Agosto, 2015. UNMSM.

Se observó que los alumnos del último ciclo de sociología consideraron algunas limitaciones en cuanto a que los profesores no habían sabido desarrollar mejor la teoría desde la cuestión empírica de los fenómenos o al revés. Por otro lado, consideraron que era necesario reestructurar el Ciclo de formación general de ciencias sociales, no sólo en cuanto al plan de estudios, sino principalmente en cuanto a la calidad pedagógica de los docentes y ayudantías en cada uno de los cursos.

La implementación de un nuevo modelo educativo por el Vicerrectorado Académico de la universidad, con la finalidad no sólo de evaluar la calidad de la educación que hasta ese momento se había venido impartiendo a los alumnos, sino proponer el tema de la enseñanza por competencias, tomó una relevancia particular. Naturalmente, este modelo educativo tenía su sustento teórico, pero el asunto estaba ¿Existen condiciones de infraestructura, como aulas, gabinetes, laboratorios, biblioteca para formar dentro del modelo teórico por competencias? ¿Es éste un modelo ideal y a la vez factual? ¿Bajo esta metodología se logrará una mejor formación profesional para la sociedad?, fueron algunas de las preguntas formuladas por los docentes en los talleres organizados por el vicerrectorado.

La universidad Nacional Mayor de San Marcos, como parte de la comunidad académica internacional, se adscribe al debate y propuestas planteadas en el seno de la UNESCO, referente a la misión y fines de la educación superior en el siglo XXI. En este aspecto, parte de la recomendación de la Comisión Internacional sobre la Educación para el Siglo XXI, dirigida por Jacques Dolors, que concibe la educación a lo largo de la vida, basada en cuatro pilares: Aprender a conocer. Aprender a hacer. Aprender a vivir juntos y Aprender a ser. Explica que hoy «importa concebir la educación como un todo. En esa concepción deben buscar inspiración y orientación las reformas educativas, tanto en la elaboración de reformas educativas, tanto en la elaboración de los programas como en la definición de las nuevas políticas pedagógicas?. Con respecto a la misión y funciones de la educación superior, la universidad asume lo planteado por la Declaración Mundial sobre la Educación Superior en el Siglo XXI: Visión y Acción de la Unesco $(1998)^{10}$.

A manera de conclusión, podemos afirmar que el tema es de difícil abordaje, pero útil para conocer de

9 Modelo Educativo San Marcos, Vicerrectorado Académico, mayo 2013.

10 Modelo Educativo San Marcos. Pág. 12, Vicerrectorado Académico. Mayo 2013. 
cómo ha sido la formación profesional de los alumnos en las diferentes etapas y contextos del escenario nacional. Asimismo, ha sido importante conversar con los alumnos del décimo ciclo, para conocer el perfil profesional con el cual egresan de las aulas universitarias y finalmente, la reflexión en cuanto a la educación por competencias dentro del nuevo modelo educativo de la universidad de San Marcos que constituye un reto tanto para las autoridades, docentes y los estudiantes. Esta nueva metodología consiste principalmente en el trabajo por proyectos, en el que a partir de una situación problema se desarrollan procesos de aprendizaje y de construcción de conocimiento, vinculados al mundo exterior, a la cotidianidad y al contexto. A todo esto, hay que agregar otro componente que es la actitud, entendido de la necesidad de una buena actitud tanto de parte del docente como del alumno que permita motivar el proceso de aprendizaje y saber convivir juntos respetando las diferencias. Esta nueva metodología se debe traducir en superar las desarticulaciones y desvinculaciones entre teoría y práctica y entre la teoría, los métodos y las técnicas de la sociología y la realidad social circundante. Esto exige a su vez un plan de estudios mediante el cual se estimule su contacto con los diferentes niveles de la realidad social, con la finalidad de que los alumnos adquieran una sólida formación personal y humanística, para el trabajo interdisciplinario y multidisciplinario, junto con valores y el saber ser como profesional.

\section{Bibliografía}

Giner, Salvador (1999). Sociología. Nueva Edición Revisada.

Giddens, Anthony (2000). En defensa de la sociologia. Traducción de Jesús Albores, Alianza Editorial.

Merton, Robert K. (1992). Teoría y estructuras sociales. México: FCE.

Salas Zapata, Walter Alfredo (7 de febrero de 2015).

Formación por competencias en educación superior. una aproximación conceptual a propósito del caso colombiano. http://www.rieoei.org/deloslectores/1036Salas.PDF.

http://ssociologos.com/2015/01/29/la-sociologiaesta-de-moda-pero-los-sociologos-no/ y datos de ISI Thomson Reuters.

Saco Alvarez, Alberto. Sociología aplicada y cambio social. ttp://ctinobar.webs.ull.es/1docencia/Cambio\%20Sociall SACO.pdf 